\title{
Controlling parameter for wave types of long flexible cable undergoing vortex-induced vibration
}

\author{
Liwu Zhang, Weimin Chen*, Zhongqin Zheng \\ Lab. of Environmental Mechanics, Institute of Mechanics, Chinese Academy of Sciences,Beijing 100190, China
}

Received 13 July 2010; revised 26 July 2010; accepted 30 July 2010

\begin{abstract}
Submerged floating tunnel (SFT) is a popular concept of crossing waterways. The failure of the cable may occur due to vortexinduced-vibration (VIV), and the stability of the cable is crucial to the safety of the entire tunnel. Investigation results in recent years show that the vortex-induced vibration of the flexible cables with large aspect ratio reveals some new phenomena, for example, the vortex-induced wave, multi-mode competition, wide band random vibration, which have brought new challenges to the study of vortex-induced vibration of long flexible cables. In this paper, the dimensionless parameter controlling the wave types of dynamic response of slender cables undergoing vortex-induced vibration is investigated by means of dimensional analysis and finite element numerical simulations. Our results indicate that there are three types of response for a slender cable, i.e. standing wave vibration, traveling wave vibration and intermediate state. Based on dimensional analysis the controlling parameter is found to be related to the system damping including fluid damping and structural damping, order number of the locked-in modes and the aspect ratio of cable. Furthermore through numerical simulations and parameter regression, the expression and the critical value of controlling parameter is presented. At last the physical meaning of the parameter is analyzed and discussed.
\end{abstract}

(C) 2010 Published by Elsevier Ltd.

Keywords: deepwater cable; lock-in; vortex-induced vibration; standing wave; traveling wave

\section{Introduction}

Submerged floating tunnel (SFT) is a potential channel floating between water surface and water bed, which provides a new option for crossing of waterways. The SFT are generally fixed by tension leg (cable), buoy and stable support. When the buoyancy is larger than gravity of SFT, tension leg (cable) is used to balance the excess buoyancy. The floating tunnel and cables often bear complicated environmental loads including wave, current and earth quake etc. When the fluid flow pass through a cable, there exist a periodic vortex shedding from the back of the cables, which produces a fluctuant lift force in across flow direction. Resonance will occur when the frequency of the vortex shedding is consistent with, or even close to the natural frequencies of the cable, vortex induced

\footnotetext{
* Corresponding author. Tel.: +86-10-82543891; fax: +86-10-82338527.

E-mail address: wmchen@imech.ac.cn
} 
vibration (VIV) is also called lock-in. The large amplitude motion of vortex-induced vibration may induce the damage of cable and especially the fluctuating stress significantly reduces the fatigue life of structure.

Most of developed prediction models for VIV such as the correlation model, statistical model and wake oscillator model are based on the assumption of standing wave vibration. But with the improving experiment technique and conditions, many large scale experiments and real field observations, of which the aspect ratio of cable even approached $10^{3}$, were implemented, some new interesting phenomena, e.g. vortex-induced wave, multi-mode vibration and wide-band random vibration are observed. So the prediction of VIV of long cables is facing new challenges.

Researches of recent years indicate that when the aspect ratio of cable is larger than $10^{3}$, the VIV often behaves as travelling wave vibration rather than standing wave vibration. Vandiver [1] did some works on the parameters important to the vortex-induced vibration of long, flexible cylinders in currents. In order to predict dynamic response of vortex-induced wave (VIW), Vandiver and Moe [2] developed the modified wake oscillator model employing assumption of infinite length of structure. Facchinetti [3] used the solution of travelling wave vibration and a phenomenological model to study the structural dynamic, hydrodynamic and the interactions between structure and fluid. In that case, before one calculate the dynamic response of a cable, which analysis model, the standing wave or travelling wave model, should be used? What is the parameter that characterizes the wave type of vibration? Moreover, besides aspect ratio is the parameter influenced by other factors? And how the expression of the parameter and its critical value are given? The work presented here aims to answer these questions.

In this paper, the response of long cables experiencing sinusoidal vortex-induced lift is studied by means of finite element simulations. Our numerical results indicate that the VIV response can be classified into three types, i.e. standing wave, traveling wave and intermediate state motion. Then dimensional analysis and parameter regression are employed to explore the parameter controlling the types of the dynamic response, or characterizing structure behavior (finite or infinite long structure). Our study indicates that the parameter is influenced by factors including system damping, aspect ratio and modal order. The parameter expression and its critical value are given. At last the physical mechanics of the parameter is discussed.

\section{Calculation model and response descriptions}

The cable is modelled as a tension beam. When lock-in occurs, the dynamic equilibrium equation of tension beam is as follow:

$$
m \frac{\partial^{2} y(z, t)}{\partial t^{2}}+\gamma \frac{\partial y(z, t)}{\partial t}-T \frac{\partial^{2} y(z, t)}{\partial z^{2}}+E I \frac{\partial^{4} y(z, t)}{\partial z^{4}}=f(z, t)
$$

where $m$ is the mass of unit length of the cable, and $\gamma$ the structural damping; $T$ is the axis tension, $f(z, t)$ is the cross-flow hydrodynamic force consists of vortex-induced lift force $f_{v}(z, t)$ and motion-induced fluid damping force $f_{f}(z, t) ; y(z, t)$ is the cylinder motion. Exact formula of hydrodynamic force $f(z, t)$ and solution of VIV is difficult to be directly derived because of the complicated interaction between fluid and structure. Here the vortexinduced lift and structure response are assumed harmonic. Then the vortex-induced lift $f(z, t)$ and fluid damping force $f(z, t)$ expressed as Morison Equation are given respectively as follows:

$$
\begin{aligned}
& f_{v}(z, t)=\frac{1}{2} C_{L} \rho V^{2} D \sin \omega_{n} t \\
& f_{f}(z, t)=\frac{1}{2} C_{D} \rho D(V-\dot{y})|V-\dot{y}|+C_{A} \frac{1}{4} \pi D^{2} \rho(\dot{V}-\ddot{y})+\frac{1}{4} \pi D^{2} \rho \dot{V}
\end{aligned}
$$

where $\omega_{n}$ is the mode frequency. $C_{L}$ is the vortex-induced lift coefficient, $C_{D}$ the drag force coefficient and $C_{A}$ the added mass coefficient, which can be determined by experiments.

The beam is assumed to have zero values of original displacement and velocity. And the boundary and original conditions of Eq. (1) are: 


$$
\begin{array}{ll}
\text { at } z=0, \quad y(0, t)=0 \text { and } \partial^{2} y(0, t) / \partial z^{2}=0 \\
\text { at } z=L, \quad y(L, t)=0 \text { and } \partial^{2} y(L, t) / \partial z^{2}=0
\end{array}
$$

when $t=0 \quad y(z, 0)=0, \quad \partial y(z, 0) / \partial t=0 \quad$ where $L$ is the length of beam. For simplification and representative, the life force is located at the middle of the beam.

A constant tension beam with a unit harmonic force applied at its center is studied, typical results of finite element simulations, the corresponding displacements RMS curves are shown in Fig. 1. It is observed that there are three types of vibration response, i.e. standing wave, travelling wave and intermediate state.

In order to estimate the wave types in a quantitative sense, here the amplitude attenuation ratios $\bar{y}_{\text {att }}$ of two in phase points are used as criteria in following form:

$$
\bar{y}_{\text {att }}=\frac{y_{1}\left(z_{1}\right)}{y_{2}\left(z_{2}\right)}\left\{\begin{array}{lr}
=1 & \text { stangding wave } \\
\geq 0.1 & \text { and }<1 \quad \text { intermediate } \\
<0.1 & \text { traveling wave }
\end{array}\right.
$$

where $z_{1}$ and $z_{2}$ are in amplitude peak points.

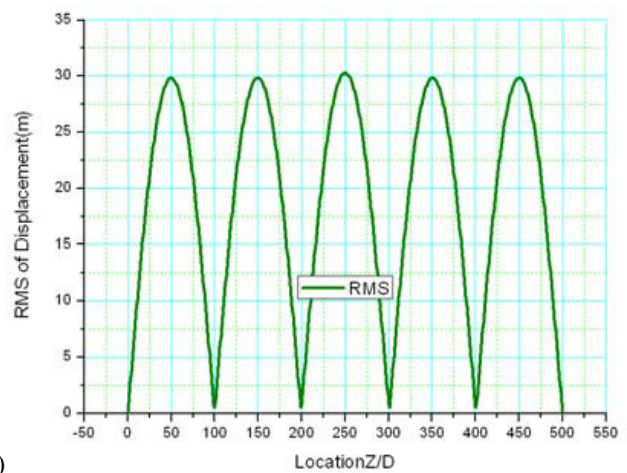

(a)

$$
\text { LocationZ/D }
$$
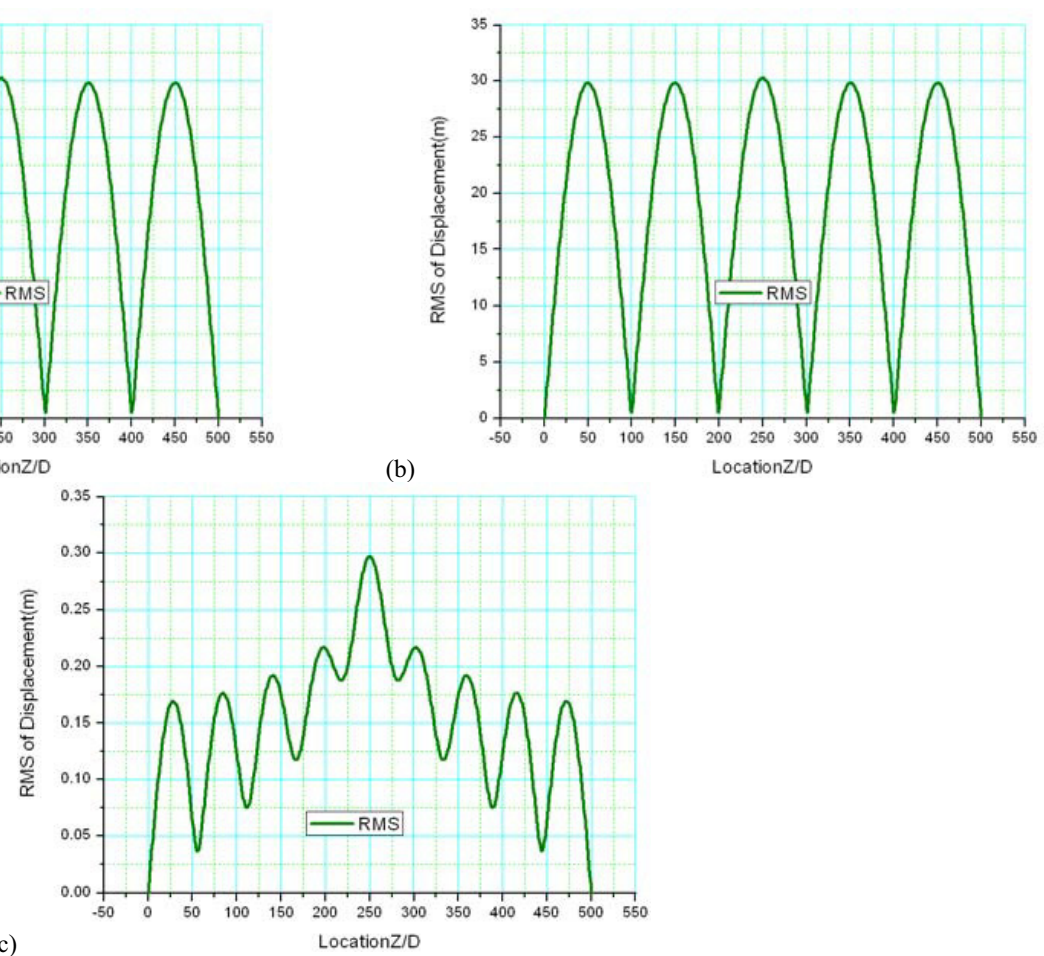

Fig. 1. Displacement RMS curves respectively for standing wave (the left plot), traveling wave (the middle plot) and intermediate state (the right plot) 


\section{The controlling parameters}

In this section, the dimensional analysis and the finite element numerical simulations are applied so as to present the expression of the controlling parameter characterizing the response wave types and subsequently give its critical value.

\subsection{Dimensional analysis}

Observing Eqs. (1)-(3), all potential factors influencing the response wave are structure and material parameters including bending stiffness $E I$, diameter $D$, length $L$, mass $m$, axis tension $T$ and damping coefficient $\gamma$, fluid parameters including flow velocity $V$, density $\rho$ and hydrodynamic coefficient $C_{A}, C_{d}$ and $C_{L}$. Then the amplitude attenuation ratios $\bar{y}_{\text {att }}$ of two in-phase points $z_{1}$ and $z_{2}$ vibrating at the $n$th mode can be expressed as:

$$
\bar{y}_{\text {att }}=\frac{y_{1}\left(z_{1}\right)}{y_{2}\left(z_{2}\right)}=f\left(E I, D, L, m, T, \gamma, V, \rho, C_{L}, C_{d}, C_{A}, \Delta z, n\right)
$$

where $\Delta z=z_{1}-z_{2}$.

Generally, for practical cable in engineering, the density $\rho$ of the fluid (seawater) and the flow velocity $V$ are already known. Besides, some structure and material parameters such as $E I, m$ and $T$ are also known. The hydrodynamic coefficient $C_{L}$ and $C_{A}$ are set to be constant in this paper. As we know, system damping more directly influences the amplitude and attenuation of dynamic response. For tension beam subjected to VIV the system damping comes from structure damping characterized by damping ratio $\zeta_{s n}$ (in our finite element simulations the structure damping is required to be input as form of mode damping ratio $\zeta_{s n}$ ) and fluid damping characterized by $C_{d}$. Additionally, $\Delta z$ can be replaced by $L\left(z_{1}\right.$ and $z_{2}$ are set to be the exciting point and the cable end, so we have $\Delta z \approx L / 2$ ). Thus Eq. (5) can be further simplified, in form of non-dimension parameter, into following form:

$$
\bar{y}_{\text {att }}=\frac{y_{1}\left(z_{1}\right)}{y_{2}\left(z_{2}\right)}=f\left(L / D, \zeta_{s n}, C_{d}, n\right)
$$

So the parameters, i.e. aspect ratio $L / D$, system damping characterized by drag coefficient $C_{D}$ and structural damping ratio $\zeta_{s n}$ and order number $n$ of excited mode, are remained as main factors influencing the cable dynamic response. Considering Eq. (4) and taking travelling wave state as turning criteria, Eq. (6) can also be rewritten as:

$$
n_{c r i}=\Pi \times f\left(L / D, C_{d}, \zeta_{s n}\right)
$$

where $n_{c r i}$ is the order number of critical mode where the dynamic response turns into traveling wave state from intermediate state. In the following sections, the influences of $L / d, C_{d}$ and $\zeta_{s n}$ on $n_{c r i}$ will be studied by numerical simulations. Consequently, an expression of the dimensionless parameter that controls the dynamic response state will be given.

\subsection{Influence of aspect ratio on $n_{\text {cri }}$}

The aspect ratio $L / D$ ranges from 800 to 5500 . The structure damping ratio and the hydrodynamic coefficient are set to be $\zeta_{s n}=0.27, C_{d}=1.2, C_{A}=1.0$ respectively. The finite element simulations are implemented to observe the vibration wave propagation behaviors. The values of $n_{c r i}$ are listed in Table 1 and are plotted against aspect ratio 
$L / D$ in Fig. 2. The polynomial fitting, parabola function (the solid line shown in Fig. 2), based on the data in Table1 is written as:

$$
n_{c r i}=23.9+5.25 \times 10^{-3}(L / D)-1.252 \times 10^{-6}(L / D)^{2}
$$

Table 1. $n_{c r i}$ and $L / D$

\begin{tabular}{lllllllll}
\hline$n_{c r i}$ & 27 & 29 & 30 & 29 & 27 & 25 & 22 & 15 \\
\hline$C_{d}$ & 800 & 1500 & 2000 & 2500 & 3500 & 4000 & 4500 & 5500 \\
\hline
\end{tabular}

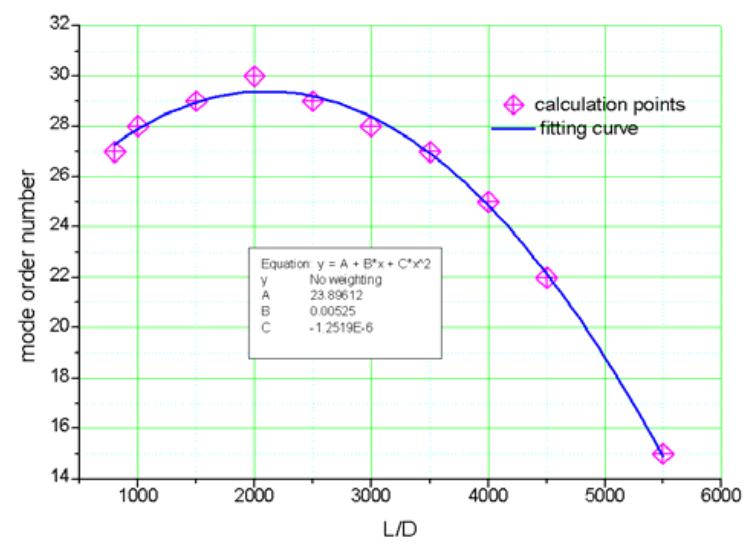

Fig. 2. Plot of $n_{c r i}$ versus $L / D$

\subsection{Influence of structure damping on $n_{c r i}$}

The mode damping ratio ranges from 0.19 to 0.9 . The aspect ratio, axis tension and hydrodynamic coefficient are set to be $L / D=1500, T=6.24 \times 10^{6}, C_{A}=1.0, C_{d}=1.2$. The value of $n_{c r i}$ are list in Table 2 and are plotted against modal damping ratio $\zeta_{s n}$ in Fig. 3. The Harris fitting formula based on data in Table 2 can be written as:

$$
n_{c r i}=\left(0.0572+0.137 \zeta^{0.304}\right)^{-1}
$$

Table 2. $n_{c r i}$ and $\xi_{s n}$

\begin{tabular}{llllllll}
\hline$n_{c r i}$ & 31 & 29 & 27 & 25 & 23 & 21 & 19 \\
\hline$\zeta_{s n}$ & 0.248 & 0.267 & 0.295 & 0.325 & 0.365 & 0.417 & 0.486 \\
\hline
\end{tabular}




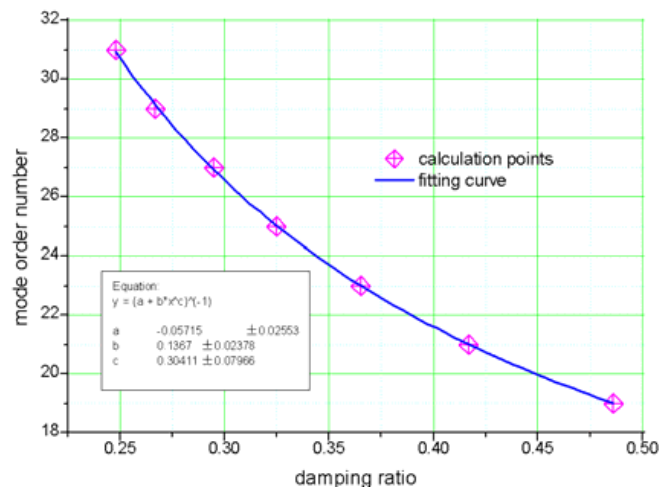

Fig. 3. Plot of $n_{c r i}$ versus $\zeta_{s n}$

\subsection{Influence of hydrodynamic drag on $n_{c r i}$}

The drag coefficient $C_{d}$ ranges from 0.02 to 5.8. The aspect ratio, mode damping ratio, axis tension and hydrodynamic coefficient are set to be $L / D=1500, \zeta_{s n}=0.27, T=6.24 \times 10^{6}, C_{A}=1.0$. The value of $n_{c r i}$ is list in Table 3 and the corresponding Harris fitting formula can be written as:

$$
n_{c r i}=\left(0.0326+0.0011 C_{d}^{1.816}\right)^{-1}
$$

Table 3. $n_{c r i}$ and $C_{d}$

\begin{tabular}{lllllllll}
\hline$n_{c r i}$ & 31 & 29 & 27 & 25 & 23 & 21 & 19 & 17 \\
\hline$C_{d}$ & 0.02 & 1.2 & 2.1 & 2.9 & 3.64 & 4.42 & 5.08 & 5.8 \\
\hline
\end{tabular}

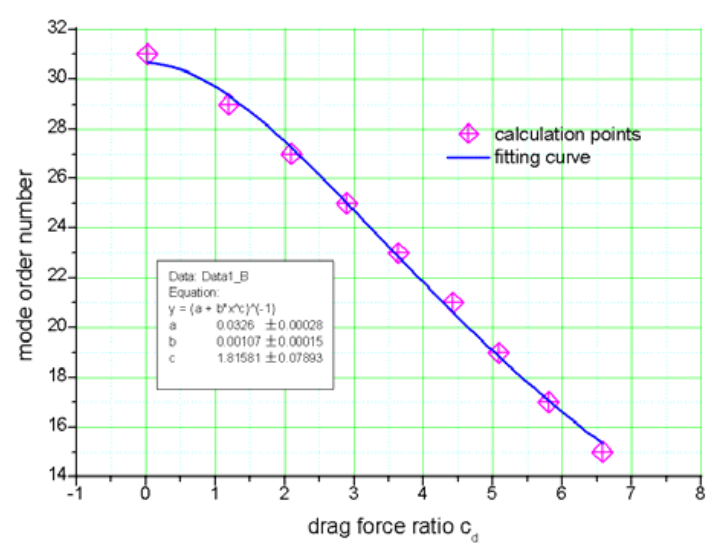

Fig. 4. Plot of $n_{c r i}$ versus $C_{d}$ 


\subsection{The dimensionless parameter and its verification}

Based on above explorations about influence of aspect ratio $L / D$, damping ratio $\zeta_{s n}$ and drag coefficient $C_{d}$ on critical mode order number $n_{c r i}$, it is assumed that:

$$
n_{c r i}=k \cdot \frac{c_{1}+b_{1}(L / D)+b_{2}(L / D)^{2}}{\left(b_{3} \zeta_{s n}{ }^{p}+b_{4}{C_{d}}^{q}+c_{2}\right)}
$$

where coefficients $k, b_{i}, c_{i}$ and power index $p, q$ can be determined by Eqs.(8)-(10) and the requirement of they satisfying self-consistent condition. For cases presented here, we have values of the coefficients as $k=0.0345$, $b_{1}=5.25 \times 10^{-3}, b_{2}=-1.25 \times 10^{-6}, b_{3}=0.137, b_{4}=0.00110, c_{1}=23.9, c_{2}=-0.0584$ and the power index $p=0.304, q=1.82$. Further, Eq. (11) can be rewritten as:

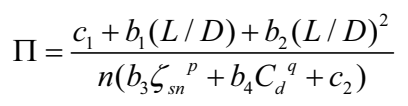

Eq. (12) indicates that the dimensionless parameter $\Pi$ can be used to characterize the wave propagation behaviors of dynamic response, and it depends on structure and fluid factors including aspect ratio $L / D$, damping ratio $\zeta_{s n}$ and hydrodynamic coefficient $C_{d}$. According to numerical results presented in this paper the critical value of $\Pi$ is given as $\Pi_{c r i}=29.0$. That is to say for a practical cable when its value of $\Pi$ is less than 29.0 , the travelling wave vibration is the dominant characteristic of cable dynamic response; when its $\Pi$ is greater than 29.0 , spatial attenuation becomes smaller and meanwhile some standing wave behavior is exhibited and tends to dominate the response with further increase of $\Pi$ values approaching to 290.

In order to verify Eq. (12), two examples are examined. For Example $1, L / D=5000, \zeta_{s n}=0.3, C_{d}=1.4, n=21$. By Eq. (12) the value of $\Pi$ is 23.2 obviously less than 29.0. That is to say the travelling wave characteristic is expected to dominate response of Example 1. For Example 2, $L / D=3000, \zeta_{s n}=0.1, C_{d}=0.6, n=19$, then we have $\Pi=106.8$. The intermediate state is expected in response of Example 2. The finite element simulation results for the two examples are respectively shown in Figs. 5 and 6. In Fig. 5 a travelling wave response can be seen from either displacement temporal-spatial evolution (Fig. 5a) or RMS curve where the attenuation ratio $\bar{y}_{\text {att }}=0.083<0.1$

(Fig. 5b). In Fig. 6 a intermediate state is seen, the attenuation ratio $\bar{y}_{\text {att }}=0.28$ is greater than 0.10 and less than 1.0 (Eq. (4)). Figs. 5 and 6 indicate that the numerical simulation results agree well with Eq. (12).

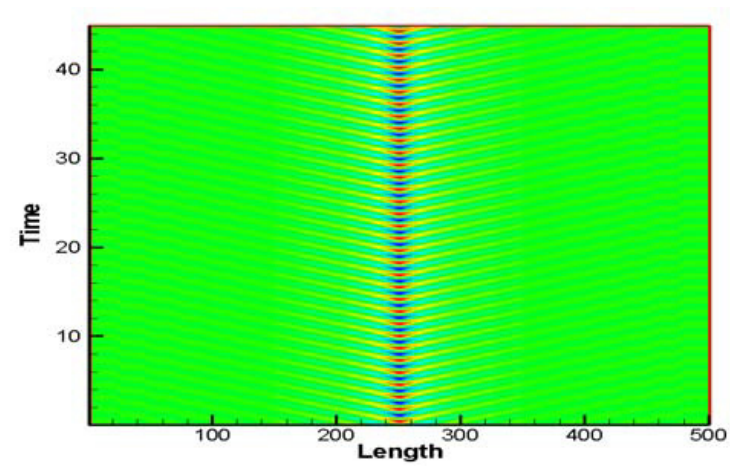

(a)

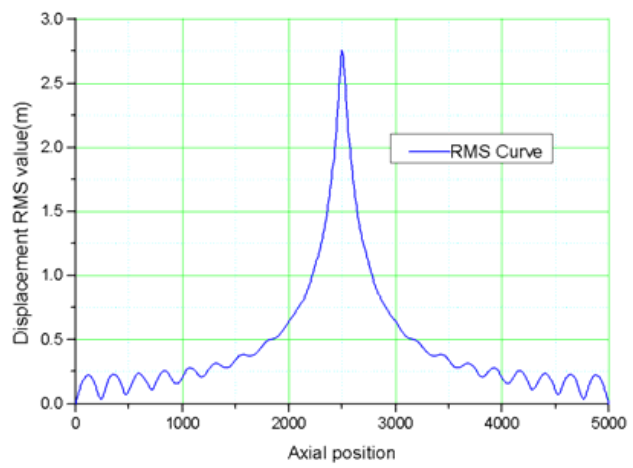

(b)

Fig. 5. Numerical results of Example 1: (a) Displacement temporal-spatial evolution; (b) Displacement RMS along span of cable 


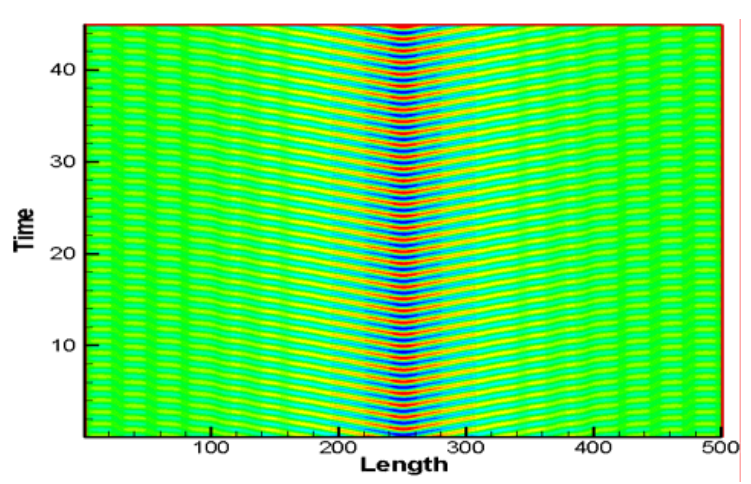

(a)

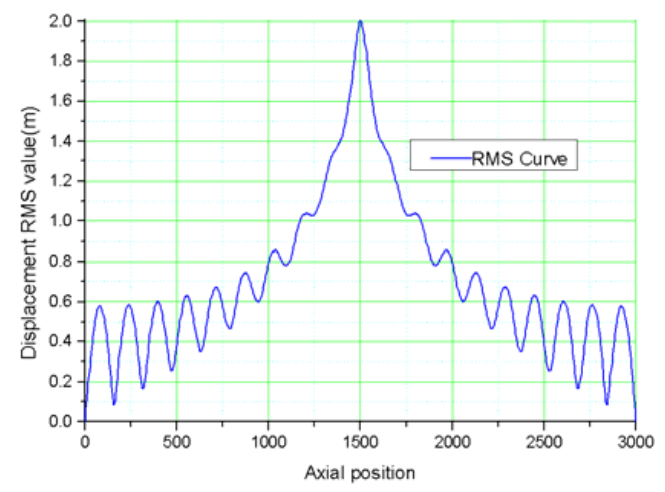

(b)

Fig. 6. Numerical results of Example 2: (a) Displacement temporal-spatial evolution; (b) Displacement RMS along span of cable

\subsection{Discussion}

The physical mechanics of the influences of parameters $\zeta_{s n}, C_{d}, n$ and $L / D$ on dimensionless parameter $\Pi$ are discussed here. Generally, when $\Pi$ becomes smaller, the cable response is more likely dominated by travelling wave.

First, the influence of structure damping ratio $\zeta_{s n}$, i.e. the lager of its value the more likely of travelling wave behaving, is understandable. When the damping ratio $\zeta_{s n}$ becomes larger, the amplitude of the structure vibration attenuates rapidly and even nearly approaches to zero so that the vibration can not arrive the structure end and reflect from the end, thus the response is dominated easily by travelling wave.

$C_{d}$ represents the damping force produced by fluid. Similarly with structure damping ratio $\zeta_{s n}$, it essentially indicates the attenuation effect, except having a nonlinear relation with vibration velocity unlike the linear relationship of structure damping, on vibration response. Thus the cable response tends to behave in a form of travelling wave when $C_{d}$ becomes larger.

Then, Eq. (12) and the calculation results (Fig. 2-4) show that the cable response is likely to be dominated by travelling wave when cable is excited at a higher order mode. The natural frequency $\omega_{n}$ of a tension beam can be written as:

$$
\omega_{n}=\frac{n^{2} \pi^{2}}{L^{2}} \sqrt{\frac{E I}{\rho A}\left(1+\frac{T L^{2}}{n^{2} \pi^{2} E I}\right)}
$$

While a wave travels along the beam, the amplitude attenuation can be described as:

$$
e^{-\zeta_{n} \omega_{n} \Delta t}=e^{-\zeta_{n} k_{n} \Delta L}=e^{-\zeta_{n} n c} \text { (for uniform beam) }
$$

where $\zeta_{n}$ is the modal damping ratio, $\Delta t$ is the propagation time of the vibration wave. Obviously, natural frequency $\omega_{n}$ has greater value for higher mode (Eq. (13)), if other parameters of the cable including $\zeta_{n}$ maintain constant, then it can be seen in Eq. (14) that the attenuation of the vibration should be larger with $n$ increasing. This means the cable response tends to behave as a travelling wave at higher excited mode.

From Eq. (11) we can see that the influence of aspect ratio $L / D$ is not monotone as that of damping $\left(\zeta_{s n}, C_{d}\right)$ and mode order number $n$. As $L / D$ increases, $\Pi$ increases first and then decreases. That is to say standing wave, 
or intermediate state, more likely dominates the cable response at small value of aspect ratio $L / D$, and then travelling wave dominates response with further increase of aspect ratio. The reason for such phenomenon may be due to the variation of system damping, produced by both structure and fluid and characterized by structure damping ratio $\zeta_{s n}$ and drag coefficient $C_{d}$, with change of aspect ratio $L / D$. The total damping of unit length can be expressed as:

$$
R=r+R_{f}\left(C_{d}\right)
$$

where $r$ and $R_{f}\left(C_{d}\right)$ is structure and hydrodynamic damping of unit length. For a uniform tension beam, structure modal damping ratio can be written as:

$$
\varsigma_{s n}=\frac{R_{s n}}{2 M_{n}} \cdot \frac{1}{\omega_{n}}=\frac{L^{2} r}{2 \pi^{2} m n^{2} \sqrt{\frac{E I}{\rho A}\left(1+\frac{T L^{2}}{n^{2} \pi^{2} E I}\right)}}
$$

When aspect ratio $L / D$ is small, structure damping $r$, greater than $R_{f}\left(C_{d}\right)$ in our finite element simulations, dominates the total system damping $R$. According to Eq. (16), the increase of beam length $L$ can result to the decrease, proportional to $1 / L^{2}$, of the structure damping $r$ if other parameters in Eq. (16) maintain constant values, the total damping force $R$ of unit length decreases fast. That means the response behaves more likely as a standing wave (see Fig. 2). As aspect ratio $L / D$ further increasing, $r$ becomes less than hydrodynamic damping $R_{f}\left(C_{d}\right)$ that is kept a constant value, then the vibration will attenuate much more because of its longer traveling distance along the cable span. That is to say the response is likely to decay before it arrive the cable end, or behave as a travelling wave.

\section{Conclusions}

In this paper, the wave characteristics of dynamic response of long flexible cable undergoing VIV are investigated. The numerical results indicate that the VIV response can be classified into three types, i.e. standing wave, traveling wave and intermediate state. By means of dimensional analysis and finite element simulations combining parameter regression, the expression and the critical value of dimensionless parameter $\Pi$ characterizing the vibration wave are given. The influences of factors $\zeta_{s n}, C_{d}, n$ and $L / D$ on $\Pi$ are discussed, and general conclusions are given as follows:

(1) As the structural damping ratio $\zeta_{s n}$ increasing, the vibration tends to attenuate rapidly, and the cable response is likely be dominated by travelling wave. The hydrodynamic drag coefficient $C_{d}$ has similar mechanics except that it has a nonlinear relationship, unlike the linear relationship of structure damping, with vibration velocity.

(2) When the cable is stimulated at modes with higher order, the attenuation of the vibration aggravates due to higher natural frequency $\omega_{n}$. The cable response is more likely behave as a travelling wave.

(3) As the cable aspect ratio $L / D$ increasing, the cable response is dominated originally by standing wave and then turns to be dominated by travelling wave because of a significant variation of total system damping consisting of structure and hydrodynamic damping.

\section{Acknowledgements}

This work is financially supported by the Knowledge Innovation Program of Chinese Academy of Sciences (Grant no. KJCX2-YW-L07) and the National Natural Science Foundation of China (Grant no. 10772183). 


\section{References}

[1] Vandiver JK. Dimensionless parameters important to the prediction of vortex-induced vibration of long, flexible cylinders in ocean currents. Journal of Fluids and Structures 1993; 7(5): 423-455.

[2] Moe G, Arntsen O. VIV analysis of cables by complex modes. 11th International Offshore and Polar Engineering Conference; 2001, p. 426-430.

[3] Facchinetti. Vortex-induced travelling waves along a cable. European J. of Mech. B/Fluids 2004; 199-208.

[4] Sarpkaya T. Vortex-induced oscillation: a selective review. Journal of Applied Mechanic 1979; 46: 241-258.

[5] Sarpkaya T. A critical review of the intrinsic nature of vortex-induced vibrations. Journal of Fluids and Structures 2004; 19: 389-447.

[6] Sarpkaya T. On the force decompositions of Lighthill and Morison. Journal of Fluids and Structures 2001; 15: 227-233.

[7] Williamson CHK, Govardhan R. Vortex-induced vibrations. Annual Reviews of Fluid Mechanics 2004; 36: 413-455.

[8] Williamson CHK, Roshko A. Vortex formation in the wake of an oscillating cylinder. Journal of Fluids and Structures 1988; 2: 355-381.

[9] Wang Y, Chen WM, Lin M. Variation of added mass and its application to the calculation of amplitude response for a circular cylinder. China Ocean Engineering 2007; 21(3): 429-439.

[10] Chen WM, Zhang LW, Li M. Prediction of vortex-induced vibration of flexible cable using an improved wake-oscillator model. Proceedings of the ASME 28th International Conference on Ocean, Offshore and Arctic Engineering, Honolulu, Hawaii; 2009.

[11] Guo HY, Fu Q, Lou M. Vortex-induced vibrations and fatigue life of marine cables conveying flowing fluid. Engineering Mechanics 2005; 22(4): 220-224. (in Chinese)

[12] Lou M. Study on the vortex-induced vibration of marine cables. Shipbuilding of China 2007; S: 369-373. (in Chinese) 